\title{
Die „Flashforward-Technik“: sich der Katastrophe stellen
}

\author{
Robin David Julian Logie \\ Chorley, Lancashire, Vereinigtes Königreich \\ Ad De Jongh \\ Universität von Amsterdam und VU Universität, Amsterdam, Niederlande
}

\begin{abstract}
Dieser Artikel stellt die „Flashforward-Technik“ vor, eine spezielle Anwendung von Eye Movement Desensitization and Reprocessing (EMDR). Sie wird zur Behandlung irrationaler Angst eingesetzt; beispielsweise wenn eine andauernde Angst auch nach Bearbeitung der Kern-Erinnerungen vergangener Erlebnisse weiterhin besteht. Der theoretische Hintergrund wird dargelegt und das Verfahren wird, auch anhand von zwei Fallbeispielen, erläutert. Wir beschreiben psychische Zustände und - Probleme, für die der Einsatz von flashforward-fokussiertem EMDR geeignet sein könnte, sowie welche Phase des therapeutischen Prozesses am besten zum Einsatz dieser Methode geeignet ist. Darüber hinaus wird die Flashforward-Technik mit anderen EMDR-Anwendungen und ähnlichen Verfahren in anderen Therapien verglichen. Einige Implikationen werden diskutiert.
\end{abstract}

Schlüsselworte: Flashforward; EMDR; Zukunftsprojektion; Erwartungsängste; katastrophische Ängste

$\mathbf{E}$ ye Movement Desensitization and Reprocessing (EMDR) wurde 1987 von Francine Shapiro zur Behandlung traumatischer Erinnerungen entwickelt (Shapiro, 2001). Seitdem entwickelte sich EMDR von einer Desensibilisierungs-Technik zu einem umfassenden psychotherapeutischen Behandlungs-Ansatz (Solomon \& Shapiro, 2008). Diese Therapie basiert auf dem Modell der Adaptiven Informationsverarbeitung (Adaptive Information Processing, AIP), welches davon ausgeht, dass psychische Störungen die Folge unverarbeiteter Informationen sind, die auf dysfunktionale Weise im Gehirn gespeichert sind ( Shapiro, 2001).

\section{Das dreistufige Protokoll}

Das Standard-Protokoll der EMDR-Therapie besteht aus einem „dreistufigen“ (Vergangenheit, Gegenwart, Zukunft) Verfahren, in dem zunächst vergangene Ereignisse, dann gegenwärtige Probleme und zuletzt voraussichtliche zukünftige Situationen im Rahmen der Therapie fokussiert werden. Die Verarbeitung vergangener Ereignisse wäre normalerweise immer der Ausgangspunkt einer Verarbeitungs-Phase in der EMDR-Therapie, und die Bearbeitung dieser Ereignisse löst üblicherweise auch bestehende psychische Probleme auf. Gemäß Shapiro (2001) führt das dreistufige Standard-Protokoll durch den gesamten Behandlungsverlauf des Patienten ${ }^{1}$. Jedes Prozessieren muss auf einen bestimmten Behandlungsfokus ausgerichtet sein. Die typische Einteilung der Behandlungsgegenstände wird im Standard-Protokoll wie folgt definiert: Zunächst werden die vergangenen pathogenen Erfahrungen bearbeitet.

Als nächstes verlagert sich der Fokus der Therapie auf die zweite Stufe, die auf die Verarbeitung spezifischer Trigger ausgerichtet ist, die gegenwärtig Beeinträchtigungen für den Patienten bewirken. Laut Shapiro (2006) können einige Trigger immernoch aktiv sein, auch wenn die ursprünglichen Traumata

This article originally appeared as Logie, R.D.J. \& De Jongh, A. (2014). The "Flashforward Procedure": Confronting the

Catastrophe. Journal of EMDR Practice and Research, 8(1), 25-32. Translated by Marlen Lenzen.

${ }^{1}$ Für einen erleichterten Lesefluss wird im Folgenden in Bezug auf den Patienten nur das Maskulinum verwendet. 
anscheinend prozessiert worden sind. Shapiro nimmt an, dass sich diese Trigger aus residualen Informationen früherer Ereignisse speisen könnten, die noch nicht vollständig verarbeitet wurden, oder dass sie durch sekundäre Konditionierung verursacht sein könnten. Diese Trigger könnten sowohl ein äußerer Umstand als auch eine innere Wahrnehmung sein (beispielsweise die Manifestation von Erwartungsangst durch Schwindel).

In der dritten Stufe der EMDR-Therapie, der Zukunftsprojektion (Future Template), hilft die Behandlung dem Patienten schließlich, einen erfolgreichen Umgang mit einem erwarteten zukünftigen Ereignis zu visualisieren. Treten beim Patienten Blockaden auf oder entsteht Unbehagen oder Angst beim Gedanken an eine zukünftige Situation, wird der Patient gemäß des Standard-Verfahrens gebeten, sich auf diese Blockaden zu konzentrieren und es werden mehrere Sets bilateraler Stimulation eingeleitet. Lösen sich die Blockaden nicht auf, empfiehlt Shapiro (2006), den Patienten mit adäquaten Informationen, Ressourcen und Fertigkeiten auszustatten, die es ihm ermöglichen, die zukünftig zu bewältigende Situation ohne Unbehagen zu visualisieren. Ebenso können der Affect Scan oder die Floatback-Technik verwendet werden, um alte Behandlungsthemen in Zusammenhang mit Blockaden, Ängsten oder Befürchtungen herauszuarbeiten. Daraufhin wird das Standard-Protokoll zur Bearbeitung dieser Themen eingesetzt. Gibt es keine erkennbaren Blockaden mehr und ,ist der Patient in der Lage, sich die zukünftige Szene mit Vertrauen und Klarheit vorzustellen" (Shapiro, 2006, S. 52), wird die dritte Stufe (die Zukunftsprojektion) installiert. Hierbei wird der Patient gebeten, das Bild, die positiven Überzeugungen und die Empfindungen $\mathrm{zu}$ fokussieren, die er mit der zukünftigen Szene verbindet; Augenbewegungs-Sets werden eingesetzt, um „sie/ ihn dabei zu unterstützen, die Informationen zu assimilieren und sie in eine positive Vorlage für zukünftige Handlungen mit einzubeziehen" (Shapiro, 2006, S. 51).

\section{Die Flashforward-Technik}

Dieser Artikel beschreibt die Flashforward-Technik; eine EMDR-Anwendung, die als Technik eingesetzt werden kann, um die irrationalen Ängste der Patienten anzusprechen, die weiterhin bestehen bleiben, nachdem die Kern-Erinnerungen vergangener Ereignisse scheinbar vollends bearbeitet wurden. In diesem Sinne kann die Flashforward-Technik als Intervention betrachtet werden, die in der zweiten Stufe (,Gegenwart") von Shapiros dreistufigem Vorgehen eingesetzt werden kann. Obwohl der Fokus des Patienten auf die Zukunft gerichtet ist, werden die durch antizipative Gedanken ausgelösten Ängste in der Gegenwart erlebt. So werden sie als aktuell bestehende Ängste betrachtet, die sich dafür eignen, in der zweiten Stufe des Protokolls bearbeitet zu werden.

Dieser Artikel beschreibt, was ein Flashforward ist und bei welchen psychischen Problemen der Einsatz von flashforward-fokussiertem EMDR angebracht sein könnte. Des Weiteren wird dargelegt, welche Phase des therapeutischen Prozesses am geeignetsten für den Einsatz dieses Verfahrens ist und es werden zwei Fallbeispiele angeführt, die das Vorgehen veranschaulichen. Abschließend wird der theoretische Hintergrund der Flashforward-Technik vorgestellt und erläutert.

\section{Forschungsbelege für die Flashforward-Technik}

Es konnte gezeigt werden, dass der Einsatz von $\mathrm{Au}-$ genbewegungen und ähnlichen ArbeitsgedächtnisAufgaben typischerweise in einer Verbesserung der emotionalen Qualität von Erinnerungen (amelioraton of the emotionality of memories) resultiert; nicht nur bei der Auflösung unbearbeiteter Erinnerungen, die einer posttraumatischen Belastungsstörung (PTBS) zugrunde liegen, sondern auch bei anderen psychischen Zuständen (De Jongh, Ernst, Marques, \& Hornsveld, 2013). Zwei kürzlich analog durchgeführte Studien haben gezeigt, dass intrusive Bilder in Bezug auf potentielle zukünftige Katastrophen ebenso entschärft werden können, indem das Arbeitsgedächtnis durch Augenbewegungen stimuliert wurde (Engelhard et al., 2011; Engelhard, van Uijen, \& van den Hout, 2010; Van den Hout et al., 2011). In beiden Studien wurden die Teilnehmer gebeten, zwei Flashforwards auszuwählen; also, negative intrusive visuelle Bilder betreffend zukünftige Ereignisse, von denen sie befürchteten, dass sie ihnen zustoßen könnten (z.B. ein Blackout während eines Vortrags, das Begräbnis eines geliebten Menschen, von einem Auto angefahren werden). Vergangene Erlebnisse wurden hierbei ausgenommen. Dann wurden die Teilnehmer zufällig zwei Gruppen zugeteilt: „Erinnerung mit Augenbewegungen“ oder „nur Erinnerung“. Daraufhin wurden vier Augenbewegungs-Sets zu je 24 Sekunden und mit jeweils einer 10-sekündigen Pause durchgeführt. Vor und nach dem Experiment wurden die Teilnehmer gebeten, das Bild abzurufen und seine Lebendigkeit und emotionale Intensität zu bewerten. Die Ergebnisse der ersten Studie $(n=28$; Engelhard et al., 2010) zeigten, dass unter der Bedingung „Erinnerung mit Augenbewegungen" die Lebendigkeit und emotionale Intensität der zukunftsorientierten Bilder 
im Vergleich zur Bedingung „nur Erinnerung“ signifikant abgenommen hatte. Die zweite Studie $(n=37$; Engelhard et al., 2011) wurde mit einer Stichprobe weiblicher Studenten durchgeführt, die auf einer Screening-Skala angegeben hatten, unter Flashforwards zu leiden. Die Ergebnisse replizierten jene der ersten Studie insofern, als dass auch hier die Lebendigkeit der Flashforwards nach der „Erinnerung mit Augenbewegungen“ im Vergleich zu „nur Erinnerung" abgenommen hatte. Ein ähnlicher Trend war in Hinsicht der emotionalen Intensität zu beobachten, jedoch reduzierte hier die „Erinnerung mit Augenbewegung " die Intensität in Prä- und Postvergleich nicht signifikant; ein Ergebnis, das die Autoren einem Problem statistischer Aussagekraft zuschrieben.

Holmes, Crane, Fennell und Williams (2007) benutzten den Begriff Flash-forwards ursprünglich in Bezug auf suizid-bezogene Bilder. Engelhard et al. (2011) beschrieben den Begriff flashforwards folgendermaßen:

. . . die Angst vor zukünftiger Gefahr ist nicht ungewöhnlich nach einem bedrohlichen Ereignis und kann in Form von zukunfts-orientierten mentalen Bildern auftreten. Diese können wie „flashforwards"erscheinen und den „flashbacks" einer posttraumatischen Belastungsstörung (PTBS) ähneln. Sie sind sinnlich-aufgeladen, lebhaft, unabweisbar und detailliert. (S. 599)

Somit bezieht sich flashforward-fokussiertes EMDR nicht auf ein erwartetes und vorhersehbares Ereignis, sondern auf die Verarbeitung eines Bildes einer befürchteten Katastrophe; sprich der mentalen Repräsentation des „Schlimmsten, was passieren könnte“ oder des „erwarteten Schreckensszenarios“. Leidet der Patient unter einer Angst, muss der Definition nach tatsächlich ein antizipiertes zukünftiges katastrophales Ereignis bestehen. Demnach sollte flashforward-fokussiertes EMDR als Intervention konzeptualisiertsein, die in derzweiten Stufe des Protokolls angewendet werden kann, da sie thematisiert, was der Patient derzeit immernoch aktiv fürchtet. Dabei sollte erwähnt werden, dass selbst, wenn die Angst in einem Ereignis gründet, das mehr als ein Jahr in der Zukunft liegt (vielleicht ein Flug oder ein Zahnarztbesuch), der Patient doch gegenwärtig mit der Angst vor diesem Ereignis belastet sein kann.

\section{Frühere Anwendungen der Flashforward-Technik}

Die einzige Veröffentlichung, die die FlashforwardTechniken in einem klinischen Kontext beschreibt, stammt von Romain (2013). Sie beschreibt in zwei Fällen die erfolgreiche Anwendung von EMDR bei Flashforwards durch den Einsatz des Standard-Protokolls. Im ersten Fall leidet eine Frau unter Ängsten bezüglich der Wiederaufnahme ihrer Arbeit, obwohl vergangene Erinnerungen erfolgreich bearbeitet wurden. Im zweiten Fall wird ein junger Mann, der erst vor kurzem seinen Alkohol-Entzug beendet hat, beim Prozessieren seiner Vergangenheit immer wieder durch Sorgen wegen eines bevorstehenden Gerichtstermins gestört.

Ein ähnliches Verfahren wie flashforwardfokussiertes EMDR wurde vor kurzem von Browning (1999) als die „Float-Forward-Technique“ beschrieben. Browning definiert diese als eine Technik, die zur Behandlung von „Blockaden, mangelnder Bereitschaft und in manchen Fällen Widerstand oder Themen im Zusammenhang mit sekundärem Krankheitsgewinn oder - Verlust" (S. 34) eingesetzt werden kann. Der Patient wird gebeten, sich ,das Schlimmste, was passieren könnte“ vorzustellen, z.B. bei der "Anwendung von EMDR", wenn sie „das Problem loswürden“, oder wenn sie "die Erwartungen ihrer Chefin an ihr Arbeitspensum begrenzen würden“. Dieses WorstCase-Szenario wird dann wie gewohnt mit dem EMDR-Standard-Protokoll prozessiert.

\section{Wann die Flashforward-Technik angewendet werden kann}

In den meisten Fällen sollte die Flashforward-Technik dann angewendet werden, wenn alle vergangenen traumatischen Ereignisse, die mit dem fraglichen zukünftigen Behandlungsgegenstand in Zusammenhang stehen, durch Einsatz des Standard-EMDR-Protokolls vollständig aufgelöst wurden. Wenn es den Anschein hat, dass der Patient immernoch Erwartungsangst bei der Konfrontation mit bestimmten Objekten oder Situationen empfindet, sollte dies den Therapeuten darauf aufmerksam machen, dass die Möglichkeit unerkannter vergangener traumatischer Ereignissen besteht, die noch bearbeitet werden müssen. Wenn alle Erinnerungen an relevante vergangene Ereignisse völlig aufgelöst wurden oder es nicht möglich ist, noch weitere vergangene Ereignisse zu identifizieren, die für das gefürchtete zukünftige Ereignis relevant erscheinen, wäre der Einsatz der Flashforward-Technik angemessen.

Ist das Flashforward gänzlich prozessiert (und ist somit das subjektive Belastungsempfinden [SUD] bezogen auf das Flashforward null) und gibt der Patient immernoch an, Unbehagen bei der zukünftigen Konfrontation mit bestimmten Stimuli oder früheren phobischen Situationen zu empfinden, oder weist er Vermeidungs-Verhalten auf, sollten andere 
Methoden eingesetzt werden. Dies würde sowohl die Zukunftsprojektion beinhalten, sowie die sogenannte Mental Video-Technik, die Teil des Phobie-Protokolls ist (Shapiro, 2001) und die Anwendung von En VivoExposition oder sogenannter behavioraler Experimente (siehe De Jongh, 2009). Der Einsatz dieser kognitiv- behavioralen Techniken kann hilfreich für den Patienten sein, sofern es als notwendig erachtet wird, dass der Patient lernt, der gefürchteten Situation ausgesetzt zu sein, bis er ein Level an Selbstbeherrschung erreicht hat, das es ihm ermöglicht, zuversichtlich mit einem gewissen $\mathrm{Ma} ß$ an Erwartungsangst und Furcht umzugehen.

Hier einige Methoden, die im Kontext einer EMDR-Behandlung angewendet werden könnten:

- Das Bearbeiten von Erinnerungen an vergangene Ereignisse, die die aktuellen Symptome des Patienten erklären

- Die Flashforward-Technik

- Die Zukunftsprojektion

- Das Abspielen eines mentalen Filmes (als Teil des EMDR-Phobie-Protokolls)

- En Vivo-Exposition / behaviorale Experimente

Dennoch gibt es hierzu ein paar Ausnahmen: Erstens sollte der Einsatz der Flashforward-Technik erwogen werden, wenn ein zukünftiges Ereignis vom Patienten so gefürchtet wird, dass es sich verheerend auf seine Lebensgestaltung auswirkt und er so entweder nicht ausreichend motiviert ist, vergangene Erlebnisse durchzuarbeiten oder nicht dazu in der Lage ist. Zweitens wäre der flashforward-fokussierte Einsatz von EMDR anzuraten, wenn es notwendig ist, einen skeptischen Patienten zunächst von den Vorteilen von EMDR zu überzeugen; hierbei kann ein aktuelles Problem bearbeitet werden, das den Patienten belastet, damit deutlich wird, dass die Verarbeitung vergangener Ereignisse der Schlüssel zum Lösen aktueller Probleme sein kann. Drittens kann es bei bestimmten Patienten nicht möglich sein, ein vergangenes Trauma oder eine negative Erfahrung herauszuarbeiten, die die Wurzel der gegenwärtigen Probleme bildet.

\section{Wie die Flashforward-Technik angewendet wird}

In ihrer Anwendung ist die Flashforward-Technik identisch mit dem Standard-EMDR-Protokoll, außer dass sich der Behandlungsfokus auf eine befürchtete zukünftige anstatt auf eine vergangene Katastrophe bezieht. Zum Beispiel würde ein Patient gefragt, der immernoch Fahrangst nach einem Verkehrsunfall empfindet, obwohl er die traumatische Erinnerung gänzlich durchgearbeitet hat, welche zukünftige Katastrophe er am meisten fürchtet. Er könnte seinen eigenen Tod in einem Verkehrsunfall antizipieren, und dieses Bild würde als Behandlungsfokus verwendet werden.

Der Therapeut kann den Patienten auffordern zu benennen, was er glaubt, ihm zustoßen könnte, wenn er nicht mehr in der Lage ist, die gefürchtete Situation zu vermeiden. Zu diesem Zweck sollte ein Rahmen geschaffen werden, der es dem Patienten erlaubt und ermöglicht, über das drohende Verhängnis des Worst-Case-Szenarios nachzudenken. Beispielsweise kann dem Patienten folgendermaßen dabei geholfen werden, sein Flashforward zu identifizieren:

Wir müssen herausfinden, was Sie befürchten bei einer zukünftigen Konfrontation mit dem, was Sie ängstigt. Was ist das Schlimmste, das Sie sich vorstellen könnten? Im Grunde suchen wir nach Ihrem ultimativen Unglücks-Szenario. Bitte machen Sie ein Standbild von diesem schlimmsten Moment.

Um die ultimative Katastrophen-Phantasie des Patienten zu identifizieren, kann der Therapeut zusätzliche hilfreiche Fragen stellen, zum Beispiel: „Was denken Sie könnte schiefgehen, wenn Sie . . [ zum Beispiel: ,einem Hund begegnen']? Wenn Sie einen Albtraum hätten . . . [zum Beispiel: ,wie Sie mit dem Auto auf einer stark befahrenen Straße zur Arbeit fahren'], wie sähe das beunruhigendste Bild dazu aus?" Es ist wichtig, dass der Therapeut das WorstCase-Szenario bis zu seiner ultimativen Konsequenz verfolgt. Zum Beispiel sollte der Therapeut, anstelle den Tod des Patienten als die ultimative Katastrophe zu akzeptieren, den Patienten fragen: „Was wäre das Schlimmste an Ihrem Tod?" Dies könnte Themen wie Verlust oder Verantwortung aufwerfen, zum Beispiel: „Meine Familie würde nicht zurechtkommen, wenn ich sterbe." Der Therapeut könnte den Patienten dann auffordern, seinen tatsächlichen Tod den angenommenen Konsequenzen seines Todes gegenüberzustellen, indem er zum Beispiel sagt: „Wenn Sie wählen müssten, was wäre jetzt am schlimmsten für Sie -zu sterben, oder am Leben zu bleiben, aber nicht für Ihre Familie sorgen zu können?" Hat der Patient schließlich ein Standbild des schlimmsten Moments seines Worst-Case-Szenarios gefunden, wird er gebeten, dieses so detailliert wie möglich zu entwerfen.

Essentielle Elemente eines für die Behandlung mit EMDR geeigneten Flashforwards

- Ein detailliertes Standbild

- Dieses beinhaltet katastrophale Elemente eines möglichen zukünftigen Ereignisses 
- Der Kontext dieses Bildes ist spezifisch und konzeptuell bezogen auf die Symptome des Patienten

- Es ist intrusiv und beunruhigend

Als Nächstes wird die negative Kognition (NK), die positive Kognition (PK), die Stimmigkeit der Kognition (validity of cognition, VOC), Gefühle, SUD und die Körperempfindung in üblicher Weise erarbeitet, um den Behandlungsgegenstand zu verarbeiten. Da der Patient generell einen Kontrollverlust erlebt, wenn er sich das Flashforward vergegenwärtigt, fühlt er sich tatsächlich machtlos gegenüber diesem - per definitionem - intrusiven Bild. Dementsprechend ist es der Erfahrung des zweiten Autors (ADJ) nach bei der Bearbeitung eines Flashforwards durch EMDR am besten, die NK ,Ich bin machtlos" als die Standard-NK zu verwenden. Dies würde ebenso bedeuten, dass ,IIch habe die Kontrolle (über das Flashforward) “ oder ,IIch kann damit umgehen (d.h. mit dem Flashforward)" als Standard-PK eingesetzt werden sollte. Daraufhin sollten alle verbleibenden Phasen des achtstufigen Standard-Protokolls (Shapiro, 2001) in üblicher Weise verwendet werden, bis ein SUD von 0 und eine VOC von 7 erreicht wurden.

Selbst, wenn alle Erinnerungen an vergangene traumatische Ereignisse im Vorhinein vollends prozessiert zu sein scheinen, kann die FlashforwardTechnik immernoch spontan altes Material aktivieren. Wenn dies geschieht (entweder als ein zuvor prozessiertes Trauma oder eine andere noch nicht verarbeitete Erinnerung), sollte der Therapeut einfach in gewohnter Weise fortfahren, zu prozessieren (d.h.: „Machen Sie damit weiter").

\section{Mögliche Anwendungen der Flashforward-Technik}

Bisher liegen noch keine klinischen Daten in Form reliabler (well-controlled) Studien zur Effektivität der Behandlung von Flashforwards vor. Nichtsdestotrotz ist die Angst vor einer zukünftigen Katastrophe eine Schlüssel-Komponente vieler psychischer Zustände. Eine Zwangsstörung (Obsessive-Compulsive Disorder, OCD) beispielsweise beinhaltet die Sorge um zukünftige Ereignisse, die der Patient als katastrophal fürchtet, und deren Vermeidung. In einer ihrer Fallstudien beschreiben Böhm und Voderholzer (2010) den Einsatz von EMDR zur Behandlung und erfolgreichen Prozessierung eines zukünftigen Szenarios, bei welchem der zwangsgestörte Patient überzeugt ist, in der Hölle bestraft zu werden.

Die Angst vor zukünftigen Ereignissen ist auch eindeutig eine Hauptkomponente bestimmter Phobien (De Jongh, ten Broeke \& Renssen, 1999; De Jongh, van den Oord \& ten Broeke, 2002). Der Einsatz von EMDR in der Behandlung von Phobien hat sich sehr bewährt, was oft die Prozessierung gefürchteter zukünftiger Ereignisse beinhaltet. Die Angst vor einer zukünftigen Katastrophe ist oft auch Merkmal anderer psychischer Störungen wie PTBS (posttraumatische Belastungsstörung; die Angst vor dem Trauma ähnlichen Situationen), Anorexie (die Angst vor den Folgen des Essens) und verschiedener Zustände, bei denen Versagensangst einen Hauptaspekt zu bilden scheint.

Situative Beispiele, in denen die FlashforwardTechnik geeignet sein könnte

Demnach birgt der Einsatz der FlashforwardTechnik Heilungspotenzial bei folgenden psychischen Zuständen - immer unter der Bedingung, dass vergangene traumatische Ereignisse oder signifikante Erfahrungen als Erstes bearbeitet werden sollten.

- Hundephobie (Angriff durch einen Hund)

- Phobien vor Zahn- und medizinischer Behandlung (extreme Schmerzen, Hilflosigkeit, „Verbluten“)

- Sozialphobie (Abgelehnt-Werden oder andere beschämende Situationen)

- Zwangsstörung (Kontaminationsängste, Hausbrand)

- Dysmorphophobie (eine negative Bemerkung zum Erscheinungsbild)

- Hypochondrie (die Endphase einer tödlichen Krankheit)

\section{Fallbeispiele}

\section{Fall Nr.1, Mary: Eine Fahrrad-Phobie}

Mary ist eine 50jährige verheiratete Frau, die durch ihre Rechtsanwälte zur Psychotherapie an den ersten Autor (RL) verwiesen wurde. Diese wurde im Rahmen einer Personenschadensklage im Zusammenhang mit einem Verkehrsunfall 18 Monate zuvor finanziert. Der Unfall geschah, als sie mit dem Fahrrad auf dem Weg zur Arbeit war; sie wurde von einem Auto angefahren und vom Fahrrad geschleudert. Zu Beginn der Therapie ist sie seit dem Unfall nicht mehr mit dem Fahrrad gefahren und hat Ängste als Beifahrerin im Auto.

Mary hat zwei Töchter im Alter von 22 und 19 Jahren, sowie einen 29-jährigen Stiefsohn aus der vorherigen Ehe ihres Mannes. Zu Ihrem Mann und zu ihren Töchtern hat sie ein gutes Verhältnis. Sie ist die zweit-älteste von vier Geschwistern; ihre Eltern blieben bis zum Tod ihres Vaters vor 11 Jahren zusammen. Sie scheint die Trauer um ihren Vater noch nicht verarbeitet zu haben und reagiert beunruhigt, wenn man sie danach fragt. Ihre Mutter ist bei guter Gesundheit und Mary hat eine gute Beziehung zu ihr. 
Mary bevorzugt es, im Auto auf dem Rücksitz mitzufahren, was zu Auseinandersetzungen mit ihren Töchtern und ihrem Mann führt. Sie vermeidet es, an ihren Unfall zu denken und wenn sie es tut, beunruhigt es sie. Ihre Fahrrad-Phobie war eindeutig durch den Unfall verursacht worden; daher war geplant, zuerst mit der Verarbeitung der Unfalls-Erinnerung zu beginnen. Nach einer Sitzung mit RessourcenInstallation dauerte es vier Sitzungen, um das Trauma gänzlich zu prozessieren. In der darauffolgenden Sitzung wurde eine Zukunftsprojektion angewendet, bei welcher sie sich vorstellen sollte, bei starkem Verkehr mit dem Fahrrad die Szene des Unfalls zu passieren. Danach antizipierte sie kaum noch Angst bei der Vorstellung, wieder Fahrrad zu fahren und erklärte sich einverstanden, dies zu versuchen. In der nächsten Sitzung berichtete sie, dass sie wieder angefangen hatte, Fahrrad zu fahren, jedoch nur bis sie ein sich näherndes Auto in einer Seitenstraße sah; dies führte zu einem Rückfall und ihr Befinden verschlechterte sich. Die Zukunftsprojektion wurde wieder eingesetzt, aber die Woche darauf sagte sie, dass sich die Dinge weiter zum Schlechteren entwickelt hätten.

Aus diesem Grund wurde entschieden, anstatt der Zukunftsprojektion nun flashforward-fokussiertes EMDR einzusetzen. Ihr Worst-Case-Szenario war, dass sie auf dem Fahrrad sterben würde (mit der NK „Ich bin verwundbar"). Die Bearbeitung stockte und verharrte bei einem SUD von 4. Darum wurde sie gefragt: „Was wäre das Schlimmste an Ihrem Tod?“. Sie sagte, dass sie ihre Familie verlieren würde, „Sie sind alle um mich; sie sind das, was ich habe. "Sie wurde dann gebeten, aus dieser Szene ein klares Bild zu erstellen, und sich hypothetisch zwischen (a) auf ihrem Fahrrad zu sterben und (b) am Leben zu bleiben, aber von ihrer Familie für immer getrennt zu sein, zu entscheiden. Sie bevorzugte die erstgenannte Option und wurde angewiesen, während der bilateralen Stimulation „mit diesem Dilemma weiter zu machen". Dies bewirkte eine dramatische Veränderung. Obwohl der SUD nicht unter 2 sank, sagte sie am Ende der Sitzung: „Wenn ich daran denke, meine Familie zu verlieren, erscheint mir Sterben jetzt als gar nicht so schlimm." Dies schien ein Wendepunkt für sie gewesen zu sein. Sie fing wieder an Fahrrad zu fahren, und zeigte eine deutliche Verbesserung der meisten ihrer Symptomen.

\section{Fall Nr. 2, Nicola: Angst vor zukünftiger Operation}

Nicola ist eine verheiratete Frau in den Dreißigern, die nach therapeutischer Hilfe suchte wegen einer
Panikstörung. Nicola hat eine Tochter im Vorschul-Alter und eine jugendlichen Stieftochter. Sie ist das einzige Kind ihrer Eltern, die sich trennten, als sie 16 war. Danach lebte sie 2 Jahre bei ihrem Vater, da sie dachte, dass ihre Mutter für die Scheidung verantwortlich sei. Sie erfuhr dann, dass ihr Vater eine Affäre gehabt hatte und entschied sich daraufhin, bei ihrer Mutter zu leben und redete 2 Jahre lang nicht mit ihrem Vater. Sie sagte dem Therapeuten, dass sie jetzt ein gutes Verhältnis zu beiden Elternteilen hätte. Sie hat eine gute Beziehung mit ihrem Ehemann, auch wenn es ihm schwerfällt zu verstehen, was sie durchmacht.

Laut Nicola liegt der Beginn ihrer Probleme zwei Jahre zurück, als sie in's Krankenhaus ging, um eine Notfall-Blinddarmoperation vornehmen zu lassen. Aufgrund einer fehlerhaften Anästhesie wachte sie im Narkose-Raum in dem Glauben auf, die Operation schon hinter sich zu haben. Sie war bewegungsunfähig, konnte jedoch hören, was gesagt wurde - einschließlich Kommentaren, dass sie "fett" sei. Während dieser sogenannten „Bewusstheits“Erfahrung hatte sie das Gefühl, nicht atmen zu können und glaubte, dass sie sterben würde. Seitdem hat Nicola jede Nacht Albträume, in denen sie das Trauma wieder durchlebt. Sie hat Panik-Attacken und Angstgefühle. Ebenso leidet sie unter Essattacken und Erbrechen, wenn sie sich besonders schlecht fühlt. Da sie nach dem Trauma begann, suizidal zu werden, kümmerte sich ihre Schwiegermutter um ihr 6 Monate altes Baby. Nicola selbst arbeitet als psychiatrische Krankenschwester in einer Akut-Psychiatrie und einige Patienten, mit denen sie gearbeitet hatte, hatten ihren Zustand verschlimmerten. Sie wurde von einem Psychiater untersucht und es wurden ihr Medikamente verschrieben; außerdem erhielt sie kognitive Verhaltenstherapie (Cognitive Behavioral Therapy, CBT) von einer Trauma-Ambulanz, aber sie hatte nicht das Gefühl, dass ihr das half.

Es wurde angenommen, dass das Trauma der 2 Jahre zurückliegenden Operation Auslöser ihrer gegenwärtigen Probleme sei, woraufhin dies als Ausgangspunkt für die Therapie genommen wurde.

In vier EMDR-Sitzungen mit dem ersten Autor (RL) wurde das Trauma gänzlich aufgelöst. Dennoch hatte sie in der letzten dieser Sitzungen eine größere Abreaktion, bei der sie realisierte, dass sie immer das Gefühl hatte, die Kontrolle haben und andere kontrollieren zu müssen. Die weitere Anamnese ergab einen Bezug zu der Zeit, als sie 16 Jahre alt war, ihre Mutter plötzlich das Haus verließ und sie anfing, sich für ihren Vater verantwortlich zu fühlen. Zwei weitere EMDR-Sitzungen $\mathrm{zu}$ diesem Ereignis und viele Diskussionen mit ihren Eltern verschafften ihr 
ein wenig Erleichterung in dieser Angelegenheit. Sie benötigte daraufhin eine weitere Sitzung, um das ursprüngliche Trauma durchzuarbeiten. An diesem Punkt bestand nur noch eine Rest-Angst vor einer weiteren Operation, die für die nahe Zukunft angesetzt war und bei der sie immernoch fürchtete, dass sich ihr Trauma wiederholen könnte.

Somit wurde eine Flashforward-Sitzung ihre größte Angst betreffend durchgeführt, nämlich dass sie während der Operation bei Bewusstsein sein und Schmerzen haben würde. Ihre NK war: „Ich bin empfindlich (I am soft). " Der SUD reduzierte sich schnell von 6 auf 1 in wenigen Sets, wobei die meisten die Wahrnehmung verschiedener Körperempfindungen beinhalteten. Sie sagte, sie wolle nicht, dass der SUD unter 1 sinke, da sie es brauche, etwas wachsam sein zu können um sicherzustellen, dass der Anästhesist beim nächsten Mal keine Fehler machen würde. Sie erkannte auch, dass es nicht möglich ist, immer die Kontrolle zu behalten und dass dies etwas ist, womit sie leben könnte.

\section{Die Flashforward-Technik aus theoretischer Perspektive}

Um zu verstehen, wie die Flashforward-Technik wirkt, ist es sinnvoll, die theoretische Perspektive der klassischen Konditionierung (Pavlov, 1927) heranzuziehen. Gemäß dieser Theorie kann ein Reiz -der konditionierte Stimulus (KS) - mit einem weiteren Reiz gepaart werden - dem unkonditionierten Stimulus (US); einem biologisch signifikanten und aversiven Reiz wie Schmerz oder einem beunruhigenden Bild. Der KS verursacht in der Regel zunächst keine besondere Reaktion; wird er jedoch mit dem US kombiniert, erzeugt er die konditionierte Reaktion (KR). Somit bezieht sich der KS zum Beispiel im Falle einer Phobie auf den Reiz, der emotionales Unbehagen hervorruft, wohingegen der US mit der Bedrohungsbewertung in Verbindung steht; also mit der Katastrophe, von der der Patient erwartet, dass sie eintreten wird und die die mentale Repräsentation der gefürchteten Konsequenz darstellt. Die Assoziation zwischen dem phobischen Stimulus und der Voraussage des Patienten (zum Beispiel dass aufgrund des Reizes wahrscheinlich ein negatives Ereignis eintreten wird), verleiht seiner Befürchtung Wirksamkeit. Der zuvor neutrale Reiz (KS), der durch klassische Konditionierung mit der Katastrophe assoziiert wurde, fängt so an, als Prädiktor der gefürchteten Katastrophe (US) zu fungieren und somit Erwartungsangst zu erzeugen. Vereinfacht ausgedrückt kann eine Person mit einer Hunde-Phobie der Überzeugung sein, dass wenn sie einem Hund zu nah kommt (KS), er sie angreifen wird (US), was eine Angst-Reaktion hervorruft (KR). Oder der Patient hat Angst vor der Situation, zur Arbeit zu fahren (KS), da er fürchtet, bei einem Verkehrsunfall um's Leben zu kommen (US), was Angst verursacht (KR). Der US wäre in diesen Fällen die imaginierte Katastrophe, angegriffen oder getötet zu werden, was als Kern des Angst-Netzwerks betrachtet werden kann.

Wenn jemand auch nach der erfolgreichen Prozessierung der vergangenheitsbezogenen KernErinnerungen immernoch ängstlich ist, ist es wahrscheinlich, dass der KS den Kern der antizipierten Gefahr triggert - die mentale Repräsentation dessen, was passieren könnte, wenn der Patient seinen phobischen Auslösern ausgesetzt ist; sprich, seinem persönlichen Unglücks-Szenario. Deswegen ist es einsichtig, dass das erfolgreiche und fokussierte Prozessieren der Vorstellung, getötet zu werden, eine fundamentale und generalisierbare Veränderung für den Patienten bewirken kann. Anders gesagt behandelt man mit flashforward-fokussiertem-EMDR nicht das, was der Patient fürchtet, sondern die Basis dieser Furcht.

\section{Unterschiede zwischen der Flashforward- Technik und der Zukunftsprojektion}

Der erste Fall ist interessant, da er Unterschiede zwischen der Zukunftsprojektion und der FlashforwardTechnik veranschaulicht. Diese Behandlung wurde zu einer Zeit durchgeführt, als der Therapeut (RL) in Bezug auf diese verschiedenen Protokolle immernoch dazulernte und mit ihnen experimentierte. Er vermutete - wahrscheinlich tun dies viele Therapeuten -, dass er die dritte Protokoll-Stufe und die Zukunftsprojektion zur Behandlung von Zukunftsangst einsetzen sollte. Jedoch linderte es Marys Angst vor dem Fahrradfahren nicht, als der Therapeut versuchte, diese mit der Zukunftsprojektion zu bearbeiten. Dies überrascht insofern nicht, als die Zukunftsprojektion darauf ausgerichtet ist, einen Plan für adaptives, positives, zukünftiges Handeln zu installieren; ihr Hauptzweck ist es nicht, Exploration zu erleichtern oder Ängste aufzulösen.

Marys Angst vor zukünftigen Ereignissen war ein Indikator dafür, dass sie immernoch unter aktuellen Triggern litt. Es zeigte sich, dass der Therapeut zur zweiten Stufe zurückgehen und die Angst bearbeiten musste, die durch Marys Katastrophen-Gedanken getriggert wurde. Die Flashforward-Technik ermöglichte es Mary, ihre Probleme auf einer grundsätzlicheren Ebene zu betrachten, als es durch die Zukunftsprojektion möglich gewesen wäre, und zu verstehen, worum es bei ihrer 
Angst wirklich ging. Indem sie zwischen der Angst zu sterben und der Angst, ihre Familie zu verlieren, differenzierte, war es möglich, das Problem neu zu betrachten. Dieser Fall zeigt deutlich, dass die Patienten durch die Bearbeitung der Probleme, die auf ,Katastrophendenken zurückzuführen sind, befähigt werden, ihre größte Angst in neuem Licht zu sehen und die diesbezüglichen ungelösten Probleme zu verarbeiten.

Insofern schließt die Flashforward-Technik die Lücke zwischen der adäquaten Bearbeitung von Erinnerungen an signifikante vergangene Ereignisse (der ersten Stufe) und der Zukunftsprojektion (der dritten Stufe). Diese Argumentation wird durch die Erfahrung des zweiten Autors (ADJ) insofern unterstützt, als dass wenn Flashforwards einmal gänzlich prozessiert wurden (SUD $=0$ und VOC $=7$ ), die Beachtung gegenwärtiger Trigger sich erübrigt. Eine weitere kritische Beobachtung ist, dass nur, wenn das Flashforward des Patienten angemessen bearbeitet wurde (aufgelöst; zweite Stufe), die Installation einer PK bzgl. eines zukünftigen Szenarios mit positivem Ergebnis (dritte Stufe) wirksam erfolgen kann.

\section{Katastrophische Ängste}

Leidet ein Patient immernoch unter irrationalen katastrophischen Vorstellung betreffend zukünftige Ereignisse, obwohl alle den Beschwerden zugrunde liegenden Erinnerungen gänzlich aufgelöst wurden, bedeutet dies, dass es immernoch dysfunktional abgespeicherte mentale Repräsentationen (unkonditionierte Stimuli, USs) gibt, die bearbeitet werden müssen. Beim Beispiel der Patientin, die Angst hatte, während einer Operation zu Bewusstsein zu kommen, kann die Beunruhigung, die mit der irrationalen Vorstellung einer zukünftigen Operation einhergeht, als „dysfunktional“ betrachtet werden. Insofern ist es realistisch anzunehmen, dass diese Vorstellung der Notwendigkeit einer Operation im Wege steht. In solchen Fällen stellt der Versuch, mit EMDR die Katastrophenbilder des Patienten anzusprechen, eine hilfreiche Vorgehensweise dar, um seine Angst zu behandeln.

Wenn ein Patient solch eine katastrophische Überzeugung äußert, kann dieses Problem folglich am besten gelöst werden, indem dem Patienten erklärt wird, dass er nicht mit der Angst, während einer Operation aus der Narkose zu erwachen, geboren wurde und seine antizipierte Angst-Situation „nur“ auf einer mentalen Repräsentation beruht; dem Überrest des früheren Traumas, das in sich selbst irrational ist und mit EMDR bearbeitet werden sollte. Es wird erhofft, dass der Patient so vom Nutzen überzeugt werden kann, sein Flashforward bis zu einem SUD von null zu prozessieren.

Ein Aspekt, der in beiden Fällen auftrat, war, dass die Patienten angaben bzgl. ihres Flashforwards mit einem SUD von über null zufrieden zu sein. Ein Therapeut könnte einwenden, dass dies auf ein unzureichend prozessiertes Flashforward hindeutet. Dies wirft eine interessante Frage auf, denn es könnte beispielsweise argumentiert werden, dass die meisten Menschen ein gewisses Maß an Unbehagen empfinden würden bei der Aussicht darauf, bei einer Operation bei Bewusstsein zu sein, Schmerzen zu haben und nicht sprechen zu können - selbst, wenn sie diese Erfahrung zuvor nie gemacht hätten. Man könnte in diesem Fall zurecht fragen, warum der SUD überhaupt bis auf null sinken sollte? Vielleicht ist es adaptiv, einen geringen Angstlevel betreffend zukünftiger Ereignisse zu haben, da dies einem ermöglicht, wachsam zu sein - in Marys Fall in Bezug auf den Gegenverkehr und in Nicolas in Bezug auf die Operationen. Möglicherwiese ist ein SUD $=1$ für Flashforwards ein gutes Ergebnis.

\section{Vergleich der Flashforward-Technik mit anderen Verfahren}

Interessanterweise wurde ein ähnlicher BehandlungsAnsatz wie die Flashforward-Technik in der CBT-Literatur zur Behandlung von Hypochondrie beschrieben (Prasko, Diveky, Grambal, Kamaradova, \& Latalova, 2010). Diese Autoren geben an, dass Patienten möglicherweise aufgrund kognitiver Vermeidung nicht das ganze Worst-Case-Szenario durcharbeiten und so weder ein Verhalten zum Umgang mit der gefürchteten Situation erarbeiten, noch sich an Katastrophengedanken gewöhnen können. Dies wiederum erhält und erhöht allmählich ihre Angst zu leiden, zu sterben und ihre Todesangst. In ihrem Modell leiten sie Patienten dazu an, sich in mehreren ExpositionsSitzungen an das Worst-Case-Szenario zu gewöhnen.

Die Flashforward-Technik hat einige Ähnlichkeiten mit Marrs (2012) Adaptiertem EMDR Phobie-Protokoll, das er zur Behandlung von Zwangs-Symptomen (Obsessive-Compulsive Disorder, OCD) entwickelt hatte. Er konzeptualisierte OCD als eine sich selbst erhaltende Störung und vermutete, dass die Zwangsvorstellungen und Zwänge, die er als gegenwärtige Trigger betrachtete, vor dem vergangenen traumatischen Ereignis angesprochen und behandelt werden müssen. Seine vorbereitende Forschungsarbeit lieferte vielversprechende Ergebnisse.

Ein weiterer Vergleich, der hier angestellt werden könnte, bezieht sich auf das „Desensitization of 
Triggers and Urge Reprocessing" (DeTUR)-Protokoll, das von Popky (2005) zum Einsatz bei Patienten mit Suchtproblemen beschrieben wird. Das DeTURProtokoll bezieht sich auch auf die Wahrnehmung eines zukünftigen Ereignisses, bei dem der Patient den Drang hat, sich in bestimmter Art und Weise zu verhalten. Allerdings fokussiert Popkys Ansatz das „Ausmaß des Drangs" zu einer bestimmten Verhaltensweise. Im Gegensatz dazu ist die Flashforward-Technik auf das Ausmaß der emotionalen Verstörung ausgerichtet, die in Bezug zu einer gefürchteten zukünftigen Katastrophe steht.

\section{Zusammenfassung}

Dieser Artikel bietet eine Anwendungs-Beschreibung der Flashforward-Technik und zeigt, wie sie in der EMDR-Therapie eingesetzt werden kann. Die zwei Fallbeispiele veranschaulichen, wie bei einigen Personen der Einsatz von flashforward-fokussiertem EMDR ein effektiver Ansatz sein kann; insbesondere beim Bestehen von Erwartungsängsten, die auch, nachdem zugrundeliegende Erinnerungen aufgelöst wurden, andauern. Wir argumentieren, dass die Flashforward-Technik sich eignet, den EMDRProzess in Situationen voranzubringen, in denen das Prozessieren vergangener Ereignisse nicht ausreicht. Wenngleich es nie als ausschließliche (,stand-alone“) Intervention betrachtet werden sollte, könnte es als ein weiteres wertvolles Instrument im Repertoire des EMDR-Therapeuten fungieren.

\section{Literatur}

Böhm, K., \& Voderholzer, U. (2010). Use of EMDR in the treatment of obsessive-compulsive disorders: A case series. Verhaltenstherapie, 20, 175-181.

Browning, C. (1999). Floatback and floatforward: Techniques for linking past, present and future. EMDRIA Newsletter, 4(3), 12, 34.

De Jongh, A. (2009). EMDR and specific fears: The phobia protocol single event trauma. In M. Luber (Ed.), Eye movement desensitization and reprocessing (EMDR) scripted protocols: Special populations (pp. 575-610). New York, NY: Springer Publishing.

De Jongh, A., Ernst, R., Marques, L., \& Hornsveld, H. (2013). The impact of eye movements and tones on disturbing memories of patients with PTSD and other mental disorders. Journal of Behavior Therapy \& Experimental Psychiatry, 44, 447-483.

De Jongh, A., ten Broeke, E., \& Renssen, M. (1999). Treatment of specific phobias with Eye Movement Desensitization and Reprocessing (EMDR): Protocol, empirical status, and conceptual issues. Journal of Anxiety Disorders, 13, 69-85.

De Jongh, A., van den Oord, H. J., \& ten Broeke, E. (2002). Efficacy of eye movement desensitization and reprocessing in the treatment of specific phobias: Four single-case studies on dental phobia. Journal of Clinical Psychology, 58, 1489-1503.

Engelhard, I., van den Hout, M., Dek, E., Giele, C., van der Wielen, J., Reijnen, M., \& van Roij, B. (2011). Reducing vividness and emotional intensity of recurrent "flashforwards" by taxing working memory: An analogue study. Journal of Anxiety Disorders, 25, 599-603.

Engelhard, I., van Uijen, S., \& van den Hout, M. (2010). The impact of taxing working memory on negative and positive memories. European Journal of Psychotraumatology, 1. http: / / dx.doi.org/10.3402/ ejpt .v1i0.5623

Holmes, E., Crane, C., Fennell, M., \& Williams, J. (2007). Imagery about suicide in depression- "Flash-forwards"? Journal of Behavior Therapy \& Experimental Psychiatry, 38, 423-434.

Marr, J. (2012). EMDR treatment of obsessive-compulsive disorder: Preliminary research. Journal of EMDR Practice and Research, 6(1), 2-15.

Pavlov, I. (1927). Conditioned reflexes: An investigation of the physiological activity of the cerebral cortex. London, United Kingdom: Oxford University Press.

Popky, A. (2005). DeTUR, an urge reduction protocol for addictions and dysfunctional behaviors. In R. Shapiro (Ed.), EMDR solutions: Pathways to healing (pp. 167-188). New York, NY: Norton.

Prasko, J., Diveky, T., Grambal, A., Kamaradova, D., \& Latalova, K. (2010). Hypochondriasis, its treatment, and exposure to the imaginative illness and death experience. Activitas Nervosa Superior Rediviva, 52, 70-76.

Romain, L. (2013). EMDR with recurrent "Flash- Forwards": Reflections on Engelhard et al.'s 2011 Study. Journal of EMDR Practice and Research, 7, 106-111.

Shapiro, F. (2001). Eye movement desensitization and reprocessing: Basic principles, protocols and procedures (2nd ed.). New York, NY: Guilford Press.

Shapiro, F. (2006). New notes on adaptive information processing with case formulation principles, forms, scripts, and worksheets. Camden, CT: EMDR Institute.

Solomon, R., \& Shapiro, F. (2008). EMDR and the adaptive information processing model. Journal of EMDR Practice and Research, 2, 315-325.

Van den Hout, M., Engelhard, I., Rijkeboer, M., Koekebakker, J., Hornsveld, H., Leer, A., . . . Akse, N. (2011). EMDR: Eye movements superior to beeps in taxing working memory and reducing vividness of recollections. Behaviour Research and Therapy, 49, 92-98.

Zuschriften zu diesem Artikel bitte an: Robin David Julian Logie, PO Box 179, Chorley, Lancashire, PR6 0GN, United Kingdom. E-mail: info@robinlogie.com 